\title{
XXX.
}

Aus dem pathologisch-anatomischen Institut zu Bern.

\section{Ein Beitrag zur Lehre von den intraabdominalen Hernien. Von}

\author{
Dr. med. O. Funkenstein, \\ Assistent am Institut.
}

(Mit 1 Abbildung.)

Unter intraabdominalen Hernien verstehen wir mit Broesike (1)*) Brüche, deren Bruchpforte in der Bauchhöhle gelegen ist, deren Bruchinhalt aber niemals aus dem Cavum abdominis heraustreten kann. Sie sind meist in Bauchfelltaschen gelegen, deren Vorkommen sehr variabel ist. Im Wesentlichen sind vier Kategorien solcher Brïche zu unterscheiden :

I. Hernien in das Foramen Winslowii.

II. Die sogenannten duodenojejunalen Hernien, deren Sitz nach Broesike fünf Bauchfelltaschen in der Umgebung der Flexura duodenojejunalis sein können.

III. Die Hernia intersigmoidea in den gleichnamigen Recessus.

IV. Die pericoecalen Hernien in Bauchfelltaschen, die am und um das Coecum herum gelegen sind.

Retroperitoneal sind von diesen Hernien im richtigen Sinne nur diejenigen zu nennen, deren Bruchsack sich in dem hinter dem Peritoneum gelegenen Bindegewebe befindet. $\mathrm{Da}$ nun über die anatomische Beschaffenheit sowie über das Vorkommen dieser inneren Hernien grosse und eingehende Monographien von J onnesco (2) und Broesike existiren, ferner die Literatur über die duodenojejunalen namentlich in letzter Zeit ziemlich angewachsen ist, ich verweise auf die Arbeiten von Abbée (4), Mánski (5), Bingel (6), so will ich mich im Wesentlichen auf das Gebiet beschränken, welches ich durch Mittheilung eines neuen Falles zu bereichern im Stande bin, nämlich auf das Gebiet der pericoecalen Hernien. In Anbetracht der Ausführlichkeit der schon erwähnten Schriften von Jonnesco und Broe-

*) Dic in Klammem befindlichen Ziffern beziehen sich auf das am Schlusse angefügte Literaturverzeichniss. 
sike kann ich hier auf eine eingehende Angabe der Literatur verzichten und dieselbe nur soweit in Betracht ziehen, als es zur Darstellung unseres jetzigen Wissenstandes von der Anatomie und Entwickelungsgeschichte der pericoecalen Bauchfelltaschen und ihren Hernien nothwendig erscheint.

Darnach ist als erster Wald eyer (6) zu nennen, welcher in seiner Habilitationsschrift zum ersten Male in zusammenfassender Weise speciell über die pericoecalen Bauchfelltaschen sich verbreitet hat. Nach ihm sind am Coecum 4 Arten von Recessus zu unterscheiden:

1. Fossa ileocoecalis superior.

2. Fossa ileocoecalis inferior.

3. Fossa coecalis.

4. Fossa subcoecalis.

Die Fossa ileocoecal. sup. kommt dadurch zu Stande, dass vom vorderen, oberen Blatt des Mesenterium ileocoecale eine meist sichelförmige, mit der Concavität nach unten und links gerichtete $\mathrm{Pe}$ ritonealfalte in schräger Richtung nach unten rechts über den oberen Einmündungswinkel des Ileum hinwegzieht und sich im vorderen Peritonealüberzug des Coecum ungefähr in dessen Mittellinie, selten am Anfang des Processus vermiformis, inserirt. Diese Falte verdankt ihren Ursprung einem in ihr verlaufenden Ast der Art. ileocolica; sie ist bald mehr oder weniger breit, bei manchen Individuen sehr fettreich, bei alten Leuten häufig nur angedeutet, bei Foeten findet sie sich schon in frühem Alter (Waldeyer konnte sie bei Foeten $5 \mathrm{~cm}$ Scheitelsteisslänge nachweisen). Während $J$ onnesco für diese Falte den Namen Repli mésentérico-coecal vorschlägt, möchte Broesike von dieser Bezeichnung deswegen abrathen, weil man darunter auch eine Falte verstehen könnte, die ab und $\mathrm{zu}$ an der Hinterseite des Coecum und von da nach dem unteren Blatt des Mesenterium laufend vorkommt. Deshalb hält er die Benennung Plica ileocoecalis anterior für die bezeichnendste. Diese Falte bildet also die vordere Wand des Recessus, seine hintere Wand stellt das Endstiick des Ileum dar. Es ist klar, dass die Tiefe des Recessus der mehr oder minder starken Ausbildung der Plica ileocoecal. ant. parallel geht. Bezüglich der Entwickelungsgeschichte der Plica ileocoecal. ant. betont Waldeyer, was auch schon Luschka (7) gefunden hat, dass nämlich in der Falte ein Ast der Art. ileocolica verläuft, welcher als ursächliches Moment für die Entstehung der Falte angesehen werden muss.

Praktisch ist diese Bauchfelltasche von geringer Bedeutung.

Von grösserer praktischer Wichtigkeit ist die zweite der Waldeyer'schen Fossae, die Fossa ileocoecal. inferior; Waldeyer 
hält sie in praktischer Beziehung für die wichtigste sämmtlicher pericoecalen Taschen. An ihr sind gleichfalls eine vordere und eine hintere Wand unterscheidbar. Die hintere Wand bildet das Mesenteriolum des Process. vermiform., die vordere Wand wird von einer Bauchfellduplikatur gebildet, welche als ein dreieckigesBlatt sich von der Vorderseite des untersten Ileumendes erhebt, den unteren Einmündungswinkel desselben in das Coecum überdeckt und sich im oberen Blatt des Mesoappendix mehr oder weniger nahe der Basis des Processus vermiformis verliert. Der freie Rand dieser Falte, die - ideale - Basis des Dreiecks verläuft bogenförmig vom Ileum mit der Concavität nach links unten zu einem in seiner Lage variabeln Punkt des Mesoappendix. Bald liegt dieser variable Punkt nahe dem freien Rande des Mesenteriolum, bald näher der Ursprungsstelle des Processus. Wechselnd ist auch seine Entfernung vom Ileum; gewöhnlich ist er von der Ansatzlinie des Mesenteriolum am Appendix nur wenige Millimeter entfernt, aber ich sah auch Fälle, wo er näher nach dem Ileum zu gerïckt war, ja sogar einen, wo er nur wenige Millimeter vom unteren Rande des Ileum entfernt blieb. $J$ e nach diesem Verhalten wechselt nun die Krümmungslinie des freien Randes, der Basis des Dreiecks, und damit dieWeite der Eingangsöffnung. Der dritte Punkt, die Spitze des Dreiecks, liegt auf der Vorderseite des Coecum, etwas oberhalb des unteren Einmündungswinkels des Ileum. Die Seiten des Dreiecks sind nun diejenigen Linien, wo sich die Duplikatur des Bauchfells von der Serosa des Darmes abhebt, resp. sich mit derselben wieder vereinigt. Die obere verläuft demnach auf dem Ileumende und entspricht ungefähr der Mittellinie dieses Darmabschnittes, die untere liegt zum grössten Theil auf dem Coecum, zum kleinsten auf dem Mesoappendix. Wald ey er schildert das Verhalten der beiden Bauchfellblätter kurz und klar wie folgt: „Der Recessus ileocoecal. inf. wird von zwei Peritonealfalten begrenzt, die den unteren Umfang des Ileumendes zwischen sich fassen und sich im Mesenteriolum des Process. vermiformis vereinigen. Die untere ist das eigentliche Mesenteriolum, die obere geht vom unteren Umfange des Ileum aus und trifft mit dem Mesenteriolum an der Basis des Processus vermiform. zusammen." Für die obere der beiden Falten hat J onnesco die glückliche Bezeichnung Repli iléoappendiculaire, und für die Tasche demgemäss den Namen Fossette iléoappendiculaire in Vorschlag gebracht, eine Bezeichnung, die wegen ihres präcisen Ausdrucks zwar von Broesike lobend angenommen wurde, aber bedauerlicher Weise dennoch bis jetzt keinen Eingang in die neueren Auflagen der anatomischen Lehrbücher und Atlanten gefunden zu haben scheint. So beharren z. B. sowohl Merkel(8) als Spalteholz (9) - der Ein- 
zige übrigens, welcher sämmtliche Recessus, mit Ausnahme eines einzigen, einer Abbildung würdigt - noch bei der alten Waldeyerschen Bezeichnung. Der Eingang zu diesem Recessus liegt, wie nach dem Gesagten wohl schon klar sein dürfte, entsprechend dem freien Rand der oberen Falte links unten, während das blinde Ende nach rechts und oben gerichtet ist.

In beiden begrenzenden Falten verlaufen nahe dem Rande und ihm parallel Gefässe, und zwar in dem Mesenteriolum die Art. appendicularis und in der Plica ileoappendicularis ein kleiner, zum Ileum zurücklaufender Zweig der gleichen $\Lambda$ rterie. Während nun die Entstehung des Mesenteriolum seit Waldeyer von allen Autoren als durch das Vorhandensein der Art. appendicular. bedingt angesehen wird, gingen die Meinungen über den Causalzusammenlang der Gefässe in der Plica ileoappendicular. mit der Entstehung dieser Falte auseinander. Und zwar deswegen, weil Lu s'chka (7) in derselben Züge von longitudinalen, glatten Muskelfasern nachgewiesen hat, die vom Coecum auf das Ileum ziehen. Toldt (10), der diese Muskelzüge schon am Ende des fünften Embryonalmonates ausgebildet fand, glaubt, dass sie in einen Zusammenhang mit der Darmmuskulatur zu bringen seien, insofern als während der embryonalen Entwickelung in dem Winkel, den der Anfang des Appendix und der Endtheil des Ileum mit einander bilden, durch „das Wachsthum beider Darmtheile nach verschiedener Richtung der Bauchfellüberzug mit einem Theile der äusseren Muskellage abgehoben wird; die so gebildete Bauchfelltasche muss sich in dem Maasse vergrössern, als die früher jenem Winkel naheliegenden Punkte des Ileum und des Wurmfortsatzes durch das fortschreitende Wachsthum von diesem sich mehr und mehr entfernen". Broesike schreibt den Muskelzügen zwar eine primäre Bedeutung für die Entstehung dieser Falte beim Embryo und bei Kindern zu, glaubt aber, dass im späteren Leben die Wachstbumszunahme derselben in Zusammenhang mit den eingeschlossenen Gefässen zu bringen sei.

Ohne über die ätiologische Bedeutung der Züge von glatter Muskulatur ein Urtheil abgeben zu können, glaube ich unter den von mir auf ihre Bauchfellverhältnisse genauer untersuchten Fällen einen anführen zu können, welcher die Bedeutung der Gefässe bei der Bildung dieser Falte auch schon im kindlichen Alter illustriren kann: Bei einem dreimonatlichen Kind von $50 \mathrm{~cm}$ Länge war die eigentliche Plica ileoappendicular. gar nicht vorhanden, wohl aber spannte sich ca. $1 / 2 \mathrm{~cm}$ oberhalb, d. h. darmaufwärts von jenem Punkt, wo sich der freie Rand des Mesenteriolum vom unteren Blatt des Mesenterium abhebt, um 
nach dem Appendix zu ziehen, eine sichelförmige Falte vom Ileum nach dem unteren Blatt des Mesenterium, und überdeckte einen kleinen Recessus, in welchen die Sonde $3 \mathrm{~mm}$ tief eindringen konnte. Der Verlauf dieser Falte war ganz ähnlich demjenigen der wahren Plica ileoappendicul., nur dass er eben nicht am Ileocoecalwinkel statt hatte, und dass statt des Mesenteriolum die Unterseite des Mesenterium zum Ansatz benutzt wurde. Die Aehnlichkeit mit der Plica appendicular. wurde dadurch noch auffälliger, dass $1-2 \mathrm{~mm}$ vom freien Rande ein feines Blutgefäss verlief. Ich möchte daher diese Falte als eine verlagerte Plica ileoappendicular. ansehen; allerdings war es mir leider aus äusseren Gründen nicht möglich, durch Injection den Zusammenhang des Randgefässes mit der Art. ileoappendicular. unzweifelhaft darzuthun.

Hernien in den Recessus ileoappendicular. gehören offenbar zu den grössten Seltenheiten, im Ganzen erwähnt Broesike aus der Literatur nur drei zweifelhafte Fälle, von denen seiner eigenen Meinung nach eigentlich nur einer, der Fall von Snow (11), mit einiger Wahrscheinlichkeit Anspruch auf Geltung machen kann. Später sind von Nasse (12) und Riese (13) je ein unzweifelhafter Fall mitgetheilt worden. Bezüglich der Anatomie der besagten Bauchfelltaschen bieten diese nichts Neues. Was die klinischen Erscheinungen anlangt, so weichen sie im Allgemeinen so wenig von dem Bilde innerer Einklemmungen $a b$, dass ich mich damit begnügen kann, auf die beiden letztgenannten Arbeiten zu verweisen.

Während die beiden bis jetzt besprochenen Recessus, resp. deren begrenzende Falten relativ constante Verhältnisse darbieten, weisen die dritte und vierte der Waldeyer'schen Fossae die grössten Variationen auf. Ein Verhalten, das sich aus ihrer Entstehungsweise ganz grut begreifen lässt. Im Gegensatz zu den beiden ersten, medial am Coecun gelegenen Fossae, die in ihrer Entwickelung vom primitiven Peritoneum abhängen, ist das Zustandekommen der lateralen und hinter dem Coecum gelegenen Recessus an mehr oder minder grosse Varietäten im Verlöthungsprocess dieses Darmtheils mit der hinteren Bauchwand gebunden. Ueber das Speciellere der Genese soll weiter unten gesprochen werden, wenn ich mitgetheilt habe, wie nach den genannten Autoren die anatomischen Verhältnisse der Fossa coecalis und subcoecalis sich verhalten.

Die zuerst von Waldeyer mit dem Namen Fossa coecalis bezeichnete Tasche entspricht dem Endtheil des Coecum, welches gewöhnlich in sie hineingesunken erscheint. Sie liegt zwischen dem Peritoneum der hinteren Bauchwand und dem Coecum. Ihre seitliche 
Begrenzung bilden 2 Peritonealfalten, von denen die eine, die laterale, in ihrem Vorkommen zienlich constant ist, während die mediale oft gár nicht oder manchmal nur unvollkommen ausgeprïgt erscheint. Die laterale Falte, seit $\mathrm{Husch}$ ke Ligamentum intestini coeci genannt, zieht vom lateralen Umfang des Coecum bald mehr von vorn, bald mehr von seitlich, bald mehr von hinten nach dem Peritoneum parietale der hinteren Bauchwand; sie endigt unten mit einem freien, bogenförmigen Rand, unter dem der Eingang zu dem Recessus gelegen ist. Das Verhalten dieser Falte zum Coecum ist nicht nur in sofern ein wechselndes, als sie bald weiter vorn, bald weiter hinten vom seitlichen Umfang abgeht, sondern auch die IIöhe des $\Lambda$ bgangspunktes d. h. desjenigen Punktes, von wo aus der freie Rand nach dem Peritoneum parietale zieht, ist eine verschiedene: bald liegt er ziemlich tief $d . h$. mehr nach dem blinden Ende des Coecum zu, bald liegt er höher oben eventuell schon auf dem Colon ascendens. Mit diesem Verhalten variirt dann selbstverständlich die Länge der Falte und die Tiefe des Recessus. Die inconstante, mediale Falte zieht vom Vereinigungswinkel des Ileum und des Coecum längs der hinteren Bauchwand auf dem M. psoas nach abwärts, Broesike nennt sie Plica infraangularis, „obwohl sie mitunter nicht genau vom Ileocoecalwinkel, sondern mehr vom linken Abschnitt des Coecum selbst abgehen kann". Wenn diese Falte nicht ausgebildet ist, wird der mediale Abschluss des Recessus von der Verlöthungslinie des Coecum mit der hinteren Bauchwand gebildet. Beide Falten, Lig. intestin. coeci und Plica infraangularis, treten manchmal zu einander in Beziehung, indem sie bogenförmig in einander übergehen und auf diese Weise eine verticale (bei liegendem Körper), halbmondförmige Falte bilden, deren Concavität nach vorne sieht, während ihr rechtes Horn am lateralen Umfang des Coecum und ihr linkes am Ileocoecalwinkel liegt. Gewissermaassen bildet dann diese Falte einen Rahmen für die untere Peripherie des Coecum und gleichzeitig eine Art Schwelle zum Eingang in die Fossa coecalis.

Ungefähr in dieser Weise ist die Fossa coecalis auf Grund der Waldeyer'schen und Broesike'schen Angaben zu schildern, die ich nach Untersuchung eines Materials von mehr als 40 Leichen nur bestätigen kann. Ueber die Art, wie Jonnesco (l. c.) jene Grube ansieht, soll weiter unten berichtet werden, wenn ich die letzte der pericoecalen Gruben, die Fossa subcoecalis - ein Name, den ibr Treitz als erster gegeben hat - in ihren Verhältnissen auseinandergesetzt habe. Sie ist in klarer Weise erst von Wald ey er beschrieben. Er fand sie tief unter dem Coecum, manchmal an der Uebergangsstelle 
in das Colon ascendens, als schmale, bisweilen doppelt angelegte Tasche des Peritoneum, die sich zwischen der hinteren Bauchwand und den genannten Darmtheilen, Coecum oder Colon asc., weit hinauf erstreckte. Ihre Eingangsöffnung war erst nach Emporheben des Coecum sichtbar, sie sah gerade nach abwärts oder ein wenig nach links, nie nach rechts. Der Verlauf dieser Grube war entweder gerade nach aufwärts oder ein wenig schräg von links unten nach rechts oben gerichtet. Als charakteristisch ist die Lage tief unter dem Coecum anzusehen.

Während nun zwar die Beschreibung bei Wal d eyer recht klar und verständlich ist, finde ich bei dem von ihm gegebenen Paradigma für diese Grube eine Stelle, die so, wie sie sich in dem mir vorliegenden Exem. plar von V. Arch. Bd. 60 S. 83 findet, ganz unverständlich erscheint. Es heisst dort wörtlich: "Neben einer ebenfalls grossen, kanalförmig ausgebildeten Fossa coecal., die den Process. vermiformis beherbergte, lag. rechts davon noch eine zweite, ähnlich gebildete Peritonealgrube am unteren Umfang des Coecum, die Fossa ileo-coecal. inf."

$\mathrm{Da} W$ aldeyer im vorhergehenden die Fossa ileo-coecal. infer. als medial vom Coecum liegend beschrieben hat und hier ein Beispiel für eine Fossa subcoecal. geben will, so kann ich es mir nicht anders erklären, als dass hier ein Druckfehler vorwaltet und es statt F. ileo-coecal. inf. F. subcoecal. heissen soll.

Was nun die Jonnesco'schen Angaben über die zwischen dem Coecum und der hinteren Bauchwand gelegenen Taschen anlangt, so unterscheidet er 2 Taschen: eine Fossette rétro-coecale externe und eine Fossette rétro-coecale interne. Die erstere soll mit der Waldeyer'schen Fossa coecalis, die letztere mit der Fossa subcoecalis von Treitz und Waldeyer identisch sein. Mit Recht weist aber Broesike nach, dass $J_{0}$ nnesco sich darin im Irrthum befindet, insofern seine Fossette rétro-coecale interne keineswegs eine Art Bett für das Coecum bildet, sondern viel eher mit der Treitz-Waldeyerschen Fossa subcoecalis in Uebereinstimmung zu bringen ist und dass hinwiederum die Fossette rêtro-coecale externe jedenfalls nicht der Fossa subcoecalis identificirt werden kann, weil nach Waldeyer's eigener Angabe deren Orificium niemals nach rechts und abwärts sieht und weil ferner bei einer Coexistenz beider Fossae niemals die Fossa subcoecalis hinter dem lateralen, und die Fossa coecalis hinter dem medialen Abschnitt des Coecum sich befinden könnte.

Für die beiden begrenzenden Falten hat $\mathrm{J}$ onn esco neue Namen gewählt, er nennt die mediale Repli mésentérico-pariétal, also diejenige, welche Broesike Plica infraangularis bezeichnet. Wir müssen jedocb 
Broesike zustimmen, wenn er seine Bezeichnung für die geeignetere hält, ebenso wie wir mit ihm einer Meinung sind, dass die von Jonnesco für die laterale Falte gebrauchte Bezeichnung, Repli pariéto-coecal oder pariéto-colique, besser als die alte Huschke'sche Bezeichnung Lig. intestin. coeci ist. Nur würden wir vorschlagen für jene Fälle, wo man diese Falte Plica parieto-colica nennen muss, wo nämlich das Coecum lateral nicht an die Bauchwand angeheftet ist, sondern die Anheftung erst oberhalb des oberen Einmündungswinkels des Ileum also am Colon ase. statt hat, dass man dann die Falte als Plica parieto-colica inferior bezeichne. Deswegen, weil weiter oben fast constant von der hinteren Bauchwand eine Duplikatur mit nach oben offenem Bogen zum Col. asc. zieht, die man als Plica parietocolica superior bezeichnen müsste. Merkwürdiger Weise findet man nirgends in der Literatur eine Bezeichnung für diese fast constant vorkommende Falte (sie fehlte unter ca. 40 von mir darauf untersuchten Fällen nur wenige Male). Gegenüber den genannten Auffassungen der Fossa subcoecalis resp. den Fossae retrocoecales vertritt Broesike eine Anschauung, welche wohl geeignet ist, einerseits das Verständniss dieser schwierigen Verhältnisse zu erleichtern, andrerseits die mannigfachen Varietäten unter einen Hut zu bringen. Er empfiehlt nämlich nur einen einzigen Recessus retrocoecalis anzunehmen, der sich hinter dem Colon eventuell weit hinauf erstrecken kann und bei entsprechend hoher Lage seines Orificium eher eigentlich einen Recessus retrocolicus darstellen würde.

Dieser Recessus liegt also zwischen der hinteren Bauchwand und der Hinterwand des Darmes (Coecum oder Colon ascend.), seine laterale Begrenzung liefert das Lig. oder die Plica parieto-coecalis resp. parieto-colica inf., die mediale Begrenzung bildet die Verlöthungslinie dieses Darmabschnittes oder des Darmgekröses mit der hinteren Bauchwand, welche Linie ,bald mehr hinter dem Mesenterium, bald hinter dem Ileocoecalwinkel, bald hinter dem Coecum oder Col.ascend. gelegen ist". Die untere Ausstrablung der Anheftungslinie stellt das Lig. infraangulare dar. Der Eingang zu dem Recess. retrocoecal. kann bald dem unteren Ende des Coecum entsprechen, bald auch höher oben hinter ihm gelegen sein, doch glaubt Broesike, dass auch Fälle möglich seien, wo die Oeffnung ganz am lateralen Theil des Recessus, also nach rechts schauend, liegen könne. Wald ey er's Fossa subcoecal. und Jonnesco's Fossette riéto-coecal externe und interne stellen nur Varietäten oder verschiedene Abschnitte des einen Recess. retrocoecalis dar. Die beiden J o nnes co'schen Gruben können, wenn sie nebeneinander vorhanden sind, ganz gut als Theile eines einzigen Recess. retrocoecal. 
betrachtet werden, die durch eine bald melur sagittale, bald mehr schräge Wand von einander getrennt sind. Ebenso kann man die F. coecal. und subcoecal. bei einer Coexistenz als einen einzigen Recessus ansehen, welcher durch ein mit einer Oeffnung versehenes, horizontales Diaphragma in einen oberen Abschnitt, Fossa subcoecalis, und einen unteren, Fossa coecal. getheilt ist. Die Waldeyer'sche Bezeichnung Fossa coecal. will Broesike beibehalten unter dem Vorbehalt, dass man darunter jene Vertiefung des Peritoneum parietale über der Fascia iliaca verstehe, in welche das Coecum eingelagert ist.

Mit dieser vereinfachten Auffassung stimmt die Genese des Recessus retrocoecal. bestens überein. Treitz schrieb dem Descensus der Sexualdrüsen einen grossen Einfluss für das Zustandekommen des Recessus zu. Aber schon Waldey er hat diese Auffassung widerlegt und dafür eine eigene Erklärung gegeben, wonach die Fossa coecal. und subcoecal. in der. Weise entstehen sollen, „dass der Blinddarm durch weiteres Wachsthum noch nach abwärts rückt, wenn bereits das Ende des Colon asc. durch Verstreichen seines Mesenterium fixirt ist; er (der Blinddarm) wird dadurch an seinen beiden Seiten wie auch am Grunde Peritonealfalten mit zwischenliegenden Taschen bilden müssen." Waldeyer sagt jedoch selbst, dass betreffs des Bildungsmodus "noch manches klar zu legen sei". In der That hat denn auch Toldt eine Erklärung für die Genese des Recessus retrocoecalis und seiner Varietäten gegeben, welche alle späteren Untersucher als klar und genügend anerkennen.

Um die Vorgänge bei der Entstehung des Recessus leichter verständlich machen zu können, muss ich auf die normale Entwicklungsgeschichte des Darmes und des Peritoneum zurückgreifen. Ich folge dabei der Darstellung, wie sie Broesike (l. c. S. 23) giebt. Darnach bildet das Mesenterium des Darmes zu einer gewissen Zeit der Entwicklung ein fächerförmiges Blatt, dessen Stiel an die hintere Bauchwand angeheftet ist, während sein freier Rand derartig von Darmschlingen umsäumt wird, dass der kurze Dickdarmabschnitt das obere Ende dieses Randes, der Dünndarmabschnitt den vorderen, unteren und hinteren Theil des letzteren einnimmt. Abgesehen von einigen Verlöthungen des Dickdarmabschnittes mit dem Duodenum, die für uns hier kein Interesse haben, bildet sich an diesem Darmtheil als erste Anheftung an die hintere Bauchwand das Lig. hepato-colicum d. i. jene Falte, welche von der hinteren Fläche der Gallenblase nach dem Duodenum und bis auf die Flexura coli dextra zieht. Von hier aus bängt das Colon ascend, und das Coecum nach abwärts. Anfänglich ist dieser Darmtheil so kurz, dass das Coecum eventuell 
dicht unter der Leber gelegen sein kann, weiterhin aber wächst es so lange nach abwärts, bis das Coecum in die Fossa il. dextr. gelangt ist. Zugleich mit der Verlängerung nach abwärts findet eine Verlöthung des freien Mesocolon ascend. mit dem Peritoneum der hinteren Bauchwand statt, deren Grenze als mediale Verlöthungslinie des Colon ascend. und des oberen Theils des Coecum erscheint. Gewöhnlich macht die Verlöthung an dieser Linie nicht Halt, sondern auch lateralwärts von dieser Verlöthungslinie legt sich die hintere Wand des Colon ascend. und des oberen Theils des Coecum an das Peritoneum an und verwächst mit demselben. Dadurch entsteht dann an dem lateralen Umfang des Colon ascend. und des Coecum an der Grenze der Verlöthung eine Linie, aus welcher später die Plica oder das Lig. parieto-coecal. hervorgeht. Es kommen aber auch Fälle vor, wo nicht nur das Coecum sondern ein beträchtlicher Theil des angrenzenden Colon ascend. völlig frei $d . h$. nicht mit dem Peritoneum parietale verlöthet ist.

Die Entstehung der retrocoecalen Bauchfelltaschen hängt nun nach Toldt allein von der mannigfach differirenden Art und Weise des Verwachsungsprocesses ab. Statt dass nämlich die Verlöthung: des Mesocolon mit der hinteren Bauchwand sich continuirlich auch auf die Hinterwand des Colon ascend. und des Coecum fortsetzt, kann sie am medialen Rand des Darmes still stehen; erst später tritt dann nur am lateralen Rand in einer von oben nach unten verlaufenden Linie die Verschmelzung mit dem Peritoneum parietale ein. Diese Verschmelzung führt zur Bildung des Lig.parieto-coecale, "dessen Entstehung und Umfang durch den Ausdehnungszustand des Blinddarms, durch gewisse geringgradige Locomotionen desselben und durch die Spannungsgrade der Bauchdecken bedingt wird". Broesike hebt hervor, dass dieses Band nicht, wie Toldt annehmen möchte, ein transitorisches ist, sondern dass seine Persistenz über jeden Zweifel erhaben sei. Was die Entstehung der Plica infraangular. und ihre Confluenz mit der Plica parieto-coecal. betrifft, so glaubt Broesike sie in einzelnen Fällen durch den Verlöthungsprocess bedingt, in anderen als in Folge einer Zugwirkung entstanden ansehen zu dürfen, wie sie durch Wachsthumslocomotionen oder Contractionen des Coecum auf ein leicht verschiebliches Peritoneum ausgeübt werden könnte. Aus einer anfänglich transitorischen Falte würde dann durch häufige Contractionen des Coecum und eventuell durch Fetteinlagerung eine persistente werden.

Dadurch, dass also die Hinterwand des Coecum oder Colon ascend. nur medial und lateral in linienförmiger Ausdehnung an die 
hintere Bauchwand angeheftet wird, kommt ein Hohlraum zu Stande, dessen Vorderwand von der (hinteren) Darmwand und dessen hintere Wand vom Peritoneum parietale gebildet wird und dessen Eingang unter dem Coecum liegt da, wo die seitlichen Anheftungen des Darmes ihr unteres Ende gefunden haben.

Eine besondere Art der Erklärung für die Entstehung der Fossa coecalis zwischen der Plica parieto-coecalis und infraangularis giebt Tar enetzki (16). Nach ihm kann man an der Fascia iliaca zwei Abschnitte unterscheiden, einen oberen, lockeren und einen unteren, festen, welch letzterer häufig mittels eines aufwärts concaven, scharfen Randes in den ersteren übergeht. Es kann nun das Bauchfell sich in den laxeren Abschnitt eventuell sogar unter den halbmondförmigen Rand hinabstülpen und auf diese Weise die Fossa coecalis bilden. Diese Vertiefung soll sowohl links als rechts vorkommen können, deshalb meint Broesike, der sie selbst nie beobachten konnte, müsse man eher den Namen Fossa iliaco-fascialis auf sie anwenden. Leider hatte auch ich nie Gelegenheit bei einer Section diese Art von Bildung der Fossa coecal. aufzufinden. Toldt scheint darin glücklicher gewesen zu sein, denn er giebt in seinem anatomischen Atlas (pag. 478) eine Abbildung einer Grube, die er allerdings als Fossa iliaco-subfascialis bezeichnet, die aber mit der Broesike'schen resp. Tarenetzki'schen Schilderung so sehr übereinstimmt, dass ich nicht anstehe, sie für identisch mit der Tarenetzki'schen Grube zu halten; sie ist in der Zeichnung links gelegen. Wenn man sich aber diese Bildung auf der rechten seite denkt und ferner in Gedanken den Blinddarm und das Colon ascend. an ihrer richtigen Stelle sich vorstellt, so kann man schon begreifen, dass der Recessus retrocoecalis eventuell auch einer solchen Bildung sein Dasein verdanken könne.

Hernien in den Recessus retrocoecalis gehören zu den seltenen Vorkommissen, hat doch Jonnesco, der mit grossem Fleisse alle nur einigermaassen in Betracht kommenden Fälle der Literatur gesammelt hat, im Ganzen nur 12 Fälle auffinden können und von diesen sind nach Broesike's kritischer Sichtung nur 4 einigermaassen anzuerkennen. In den letzten 10 Jahren ist, soweit ich finden konnte, nur ein neuer, zuverlässiger Fall von $\Lambda$ schoff (17) mitgetheilt worden.

Bevor ich jedoch auf die Casuistik selbst eingehe, möchte ich sowohl über die Genese der retrocoecalen Hernien als auch über die an sie zu stellenden charakteristischen Anforderungen einiges vorausschicken.

Was das Zustandekommen der Hernia retrocoecalis anlangt, so 
stellt Jonnesco folgende Theorie auf : das Coecum legt sich nach dem Aufhören einer energischen Contraction vor eine der binteren Bauchwand dicht anliegende Dünndarmschlinge, anstatt dieselbe bei Seite zu schieben. Durch einen plötzlichen Druck auf die Bauchlıöhle z. B. bei einer starken Beugung soll dann die Dünndarmschlinge in den offenen Recessus retrocoecalis hineingetrieben werden.

Während Aschoff in seinem Falle eine Bestätigung der J on nesco'schen Theorie zu finden glaubt, tritt Broesike dieser Auffassung von der Genese der Hernie deshalb entgegen, weil nach seiner Meinung die Bauchpresse auf alle Eingeweide gleichmässig wirken müsse und nicht einzusehen sei, weshalb dieselbe gerade eine bestimmte hinter dem Coecum gelegene Dünndarmschlinge in der Richtung von unten nach oben in die Höhe drängen soll. Die Bauchpresse müsste lediglich das Coecum gegen die Dünndarmschlinge und beide Darmtheile zusammen gegen die hintere Bauchwand drücken. Nach Broesike's eigener Meinung sind zum Zustandekommen einer Hernie in den Recessus retrocoecalis zunächst solche Momente nothwendig, die eine Dislocation des blinden Coecalendes nach vorn und oben bewirken, i. e. starke Anfüllung des Coecum, eventuell Adhäsionen seiner vorderen Wand mit einem benachbarten beweglichen Eingeweide u. s. w. Dadurch ist den Dünndarmschlingen, namentlich wenn sie ein langes Mesenterium haben, Gelegenheit zur Interposition zwischen Coecum und hintere Bauchwand gegeben; eine solche ist aber an und für sich noch keineswegs als Hernie anzusehen, erst wenn eine Dünndarmschlinge durch das Orificium des Recessus eingetreten ist und von demselben festgehalten wird, wobei also eine gewisse Enge des Ringes vorausgesetzt werden muss, sind sämmtliche Anforderungen, die man an eine Hernie zu stellen hat, als erfüllt anzusehen. Welcher Art die Gewalt ist, welche den Darm in den Schnürring hineintreibt, ist bis jetzt noch unerklärlich.

Als charakteristisches Merkmal für eine Hernia retrocoecalis ist an erster Stelle das anzusehen, dass die Bruchpforte zum Theil vom Coecum und der hinteren Bauchwand gebildet wird; weiter ist bei kleinen und mittelgrossen Brüchen ihre Lage hinter dem Coecum charakteristisch. Bei grossen Brüchen können diese besonderen Merkmale verwischt werden, hauptsächlich deshalb, weil bei starker Ausdehnung des Bruchsackes eine Wanderung der Bruchpforte eintreten kann. Diese Wanderung könnte natürlich in Anbetracht der Schwere des Bruchinhaltes und der aufrechten Körperhaltung nur nach abwärts stattfinden.

Was nun die Casuistik der retrocoecalen Hernien anlangt, so ist 
gleich hier zu betonen, dass alle jene Fälle, die Jon nesco zusammengestellt hat, unter einer mangelhaften Darstellung leiden, selbst die vier, von Broesike einigermaassen anerkannten Fälle zeigen diesen Mangel. Da auch uns die Gründe, welche Broesike veranlassten, von den Jonnesco'schen Fällen 8 als jedenfalls nicht zu den retrocoecalen Hernien gehörig zu streichen, völlig beweiskräftig erscheinen, so dürfte es genügen, hier nur jene 4 Fälle anzuführen, die mit einiger Wahrscheinlichkeit als wahre Hernien in den Recessus retrocoecalis anzusehen sind. Drei von ihnen hat Rieux (18) veröffentlicht, der vierte stammt von Engel (19).

Der erste Fall von Rieux zeigte ein $8 \mathrm{~cm}$ langes, eingeklemmtes Dünndarmstück in einer unter dem Coecum gelegenen Cavität.-Die blindsackförmig endende Höhle scheint eine quere Lage eingenommen zu haben. Sie war an ihrer oberen wie an ihrer unteren Parthie ganz von glattem Peritoneum austapezirt, ähnlich demjenigen, welches die obere Fläche des Coecum bekleidet. Ueber die Bruchpforte äussert sich der Autor: "Autour de l'entrée de la cavité éxiste un épaississement du tissu cellulaire, souspéritonéal, représenté par un relief circulaire $d u$ péritoine, et si, a quelques centimètres de distance, on exerce une traction sur le péritoine, ce relief forme des lieus qui ferment complètement la cavité à la manière des cordons de bourse." Man ist nicht sicher, ob diese Stränge nicht 'etwa Zeichen einer alten Peritonitis darstellen und ob nicht vielleicht die ganze "abnorme" Höhle als Folge abgelaufener peritonitischer Processe anzusehen ist.

Im zweiten Fall von Rieux fand sich ein $5 \mathrm{~cm}$ langes, stark comprimirtes Dünndarmstück in einer Höhle hinter dem Coecum. Was der Autor über die Höhle sagt, ist recht dürftig: „une sorte de cavité doublée par le péritoine, fermée en bas par le péritoine épanoui et formant une bride péritonéale bien nette et dans un autre sens par le coecum lui-même. Cette cavité peut recevoir la moitié de la longueur du petit doigt."

Wenn auch Zweifel bestehen können, ob diese Höhle nicht etwa gleichfalls durch peritonitische Processe entstanden sein könne, so scheint es doch wahrscheinlicher, dass sie unserem Recessus retrocoecalis entspricht.

Der 3. Fall ist noch kürzer abgethan. Rieux giebt nur an, dass ein 4-5 cm langes, leicht bewegliches Dünndarmstück unter dem Coecum in eine abnorme Höhle eingedrungen war, die ganz von Peritoneum ausgekleidet war und bei welcher der Rand der Eintrittsöffnung eine leichte Erhabenheit bildete.

Alles in allem stellen diese 3 Fälle möglicher Weise, vielleicht Deutsche Zeitschrift f. Chirurgie. LXIV. Bd. 
sogar wahrscheinlich, Hernien des Recessus retrocoecalis dar, mit voller Sicherheit lässt sich das jedoch nicht behaupten.

Der Fall von Engel zeigt eine innere Hernie, die nach Annahme des Autors durch Eintritt des grössten Theiles der Dünndarmschlingen in die von ihm sogen. "Blinddarmtasche" entstanden ist. Ohne Zweifel ist diese Tasche mit dem Recessus retrocoecalis identisch. Der Bruchsack lag retroperitoneal, nahm hauptsächlich die rechte Bauchhälfte ein und communicirte durch eine 2 Zoll breite Bruchpforte mit dem Cavum peritonei. Der Bruchinhalt bestand aus dem ganzen Dünndarm, ausgenommen dem obersten Theile des Jejunum und dem untersten Ende des Ileum. Das Coecum lag oberhalb und etwas links vom Nabel, Grimmdarm und S. romanum füllten die linke Unterbauchgegend aus. Peritonitische Erscheinungen fehlten. Ueber Lage und Begrenzung der Bruchpforte giebt Engel weiter nichts an. Broesike meint, wenn man den Fall als grosse Hernie des Recessus retrocoecalis ansehen wolle, so müsse man auf die Autorität von Engel hin glauben, dass es so gewesen ist, lediglich nach der Beschreibung könne er nur als intraabdominale Hernie gelten.

Der von Asch off vor 6 Jahren publicirte Fall betraf eine 48 . jährige Frau, die 8 Geburten in kurzen Abständen durchgemacht hatte und seit der letzten Entbindung stark abgemagert war. Sie verspürte beim Bodenscheuern plötzliche heftige Schmerzen in der rechten Unterbauchgegend gerade in dem Moment, als sie sich aus der gebeugten Stellung aufrichten wollte. Tags darauf begannen Erbrechen, Ohnmachtsanfälle und Stuhlverhaltung. Am 21. Krankheitstage wurde die Kranke zur Operation ins Krankenhaus geschickt. Bei der Untersuchung erwies sich der Leib weich, sehr stark aufgetrieben, links mehr als rechts. Die Diagnose lautete auf chronischen Darmverschluss, angesichts des kachektischen Aussehens der Patientin wahrscheinlich als Folge einer carcinomatösen Strictur, deren Sitz in der linken Flexura coli angenommen wurde. Zwecks Anlegung eines Anus praeternatural. wurde über der Coecalgegend der Bauch aufgeschnitten. Es zeigte sich der Dünndarm stark geröthet und aufgetrieben, er uberlagerte das Colon. Dieses letztere war stark collabirt, ebenso war die Einmündungsstelle des Ileum ins Coecum eng und leer. Die zusammengefallenen Ileumschlingen liessen sich in eine Tasche verfolgen, die hinter dem Coecum und dem Colon ascend. lag und ganz mit collabirten Dünndarmschlingen angefültt war. Unter Zerreissung einiger Adhäsionen, die sich zwischen den Darmschlingen untereinander und auch von diesen zur Höhlenwand ziehend vorfanden, wurde der Darm aus der Tasche hervorgezogen. Der Eingang zur Tasche wurde ein- 
gekerbt, worauf es möglich war, die Hand einzuführen und zu constatiren, dass die geräumige Höhle hinter dem Coecum gelegen und medial begrenzt wurde vom Mesenterium des Coecum und des Colon ascend., dass die Hinterwand aus dem Peritoneum parietale bestand und dass nicht etwa noch ein anderer Bruchsack vorhanden war. Am zuführenden Schenkel des Dünndarms fand sich eine Schnürfurche.

Ohne Zweifel ist Asch off völlig im Recht, wenn er seinen Fall als richtige Hernie des Recessus retrocoecalis ansieht. Sie hat die Besonderheit, dass sie die erste zuverlässige Beobachtung einer grossen Hernie in diesen Recessus darstellt und dass sie geeignet scheint, die Jonnesco'sche Theorie von der Genese solcher Hernien zu stützen. Die Patientin hatte in kurzer Zeit hintereinander mehrere Geburten durchgemacht, war stark abgemagert - Momente, deren prädisponirender Einfluss für die Entstehung solcher Hernien auch von Broesike zugegeben wird -, im Moment des Eintritts der Hernie befand sich die Patientin in einer Stellung, in welcher der Darm nach vorn und unten gefallen und der Eingang zu dem Recessus weit geöffnet sein musste, jetzt wurden durch eine starke Wirkung der Bauchpresse die Dünndarmschlingen in den Recessus hineingepresst.

Diesen Fällen möchte ich nun den meinen unter Vorausschickung einiger klinischer Daten anreihen.

Die klinischen Angaben wurden mir durch die Güte des Herrn Prof. Girard zu Theil, dem ich an dieser Stelle meinen besten Dank ausspreche. Herr Prof. Girard wurde zu der Patientin am 26. März 1902, dem Tage der Operation, als Consiliarius zum ersten Male gerufen. Anamnestisch wurde inm bekannt, dass die Patientin vor Kurzem ein schweres Erysipelas faciei uberstanden hatte, dessen Spuren noch an der sich schullfernden Haut des Gesichts festzustellen waren. Die jetzige Krankheit habe vor 5 Tagen mit kolikartigen Schmerzen im Abdomen eingesetzt, dieselben seien im weiteren Verlauf immer heftiger aufgetreten und seien bald von Kothbrechen begleitet worden. Stuhlgang sei zuletzt vor 3 Tagen in geringer Menge abgegangen. In den ersten Tagen der Erkrankung sei besonders die rechte Unterbauchgegend auf Palpation schmerzhaft gewesen. Als Herr Prof. Girard die Kranke untersuchte, war der ganze Leib stark aufgetrieben und uberall gleichmässig schmerzhaft. In Anbetracht der offenkundigen Zeichen eines acuten Darmverschlusses und Angesichts des Mangels einer nachweisbaren Inguinal- oder Cruralhernie dachte man an eine innere, eventl. durch Verwachsungen in der Gegend des Processus vermiformis verursachte Incarceration und schritt demgemäss alsbald zur Operation. Nach der Eröffnung der Bauchhöhle griff Herr Prof. Girard sofort durch das Convolut der stark geblähten Dünndärme nach der rechten Unterbauchgegend und konnte dort auch thatsächlich fast sofort einen derben Ring fúthlen, in den eine Darmschlinge eingeklemmt 
war. Die Lösung liess sich rasch und ohne Mühe bewerkstelligen. Inzwischen hatten sich aber bei der Patientin sowobl drohende Symptome von Seiten des Pulses, als auch eine starke Cyanose des Gesichts und zugleich heftiges Erbrechen eingestellt, sodass man von einer weiteren Orientirung uber die Art und Lage der Bruchpforte absehen und schleunigst die Operation beenden musste. Das Erbrechen dauerte jedoch fort und nach 2 Stunden trat bei der Patientin der Exitus ein.

Bei der am nächsten Tage, 27. März 1902, erfolgten Section konnte ich folgenden Befund constatiren:

Mittelgrosser Körper in gutem Ernährungszustand. Rechte Gesichtshälfte zeigt stark blaurothe Farbe, iiber ihr schuppt sich an manchen Stellen die Epidermis in kleinen Fetzen ab. $3 \mathrm{~cm}$ oberbalb der Incisura jugular. sterni eine frisehe Tracheotomiewunde, aus welcher röthlicher Schaum hervorkommt. In der Mittellinie des Bauches, $1 \mathrm{~cm}$ oberhalb des Nabels beginnend und im Ganzen $17 \mathrm{~cm}$ lang eine durch Nähte verschlossene Laparotomiewunde, die nach Lösung der Nähte weit klafft; die Wundränder glatt, ohne Reaction.

Bauchsitus: Zwerchfell beiderseits in Höhe der 5. Rippe.

Leberrand in der Mammillarlinie in Höhe des Rippenbogens, er läuft demselben parallel nach links. Linkerseits kommt der Magen in handbreiter Ausdehnung stark gebläht unter der Leber hervor. Auch die Dünndärme sind stark gebläht. ihre Serosa ist feucht und mit einer ganz dünnen Schicht röthlicher Flilssigkeit bedeckt. An einer Dünndarmschlinge ist die Serosa in geringer Ausdehnung matt und trocken, leicht geröthet und von einem feinen Fibrinbelag bedeckt; hier zeigt der Darm eine seichte circuläre Einschntirung von $1-2 \mathrm{~mm}$ Breite. Die Serosa dieses Schnurringes ist ziemlich stark geröthet. Wie sich später feststellen lässt, beträgt die Entfernung dieser Stelle von der Baulini'schen Klappe ca. $3 \mathrm{~m}$. Ungefähr $25 \mathrm{~cm}$ oberhalb dieses Ringes findet sich eine feine Andeutung einer zweiten circulären Finschnürung, hier ist jedoch die Serosa fast kaum eingezogen und in ihrer Farbe eher blasser als die gewöhnliche Darmserosa. Das den beiden Schnürringen entsprechende Mesenterium weist mehrfache punktförmige Röthungen auf, jedoch nur in zwei kleinen liezirken.

In der Bauchhöhle befinden sich ca. 50-60 cem röthliche, klare Flussigkeit. Das Peritoneum parietale und viscerale, ausgenommen die genannte Stelle, uberall glatt und glänzend und ohne Röthung.

Das Mesenterium der Dünndärme erscheint ziemlich lang, es misst von der Radix mesent. bis zum Darmansatz $21 \mathrm{~cm}$.

Der Recessus duodenjejunal. wird von einer oberen Falte, der Plica duodeno-mesenterica, gebildet, er vermag das Endglied des Zeigefingers bequem aufzunehmen.

Schlägt man das stark ausgedehnte und aufgeblähte Coecum etwas nach oben zurück, so sieht man unter seinem lateralen Rand eine schwach begrenzte runde, $2 \mathrm{~cm}$ im Durchmesser betragende Oeffnung, die von einem Ring stark gerötheter Serosa gebildet wird (cf. Abbild. R). Durch sie gelangt der Finger in eine $8 \mathrm{~cm}$ lange Höhle, deren Lumen weiter als der ringförmige Eingang ist und deren Wand ringsum aus glattem Peritoneum zu bestehen scheint. Sie verläuft in ganzer Ausdehnung hinter 
und unter dem Coecum, zum Theil auch noch unter dem Colon ascendens, da ihr blindes Ende höher als der obere Einmündungswinkel des Ileum gelegen ist. Die Axe der Höhle ist leicht schräg nach rechts oben und aussen gerichtet.

Das Colon ascend. ist mit einem kleinen Mesocolon an der hinteren Bauchwand angeheftet, an der Verlöthungslinie desselben mit dem Peritoneum parietale befinden sich vielfache kleine Falten, die bald nach oben, bald nach unten convex kleine Gruben und Recessus von $3-5 \mathrm{~mm}$ Tiefe bedecken. Sie sind auch weiter unten an der seitlichen Anheftungslinie des Coecum vorhanden.

Die Plica ileocoecal. ant. ist kurz, sehr fettreich, der darrunterliegende Recessus nur angedeutet. Auch die Plica ileoappendicularis (Pl. il. a. $d$. Abbildung, ist sehr fettreich, sie ist ziemlich breit, bis zu $2 \mathrm{~cm}$, ihre Insertionsstelle an dem ebenfalls sehr fettreichen Mesenteriolum ent. spricht ungefähr dem Anfang des zweiten Drittels des Processus vermiformis. Der letztere zeigt keinerlei Veränderungen, er ist ca. $6 \mathrm{~cm}$ lang. Un. gefähr $2 \mathrm{~cm}$ vor seinem Ende erhebt sich im vorderen oberen Blatt des Mesenteri-

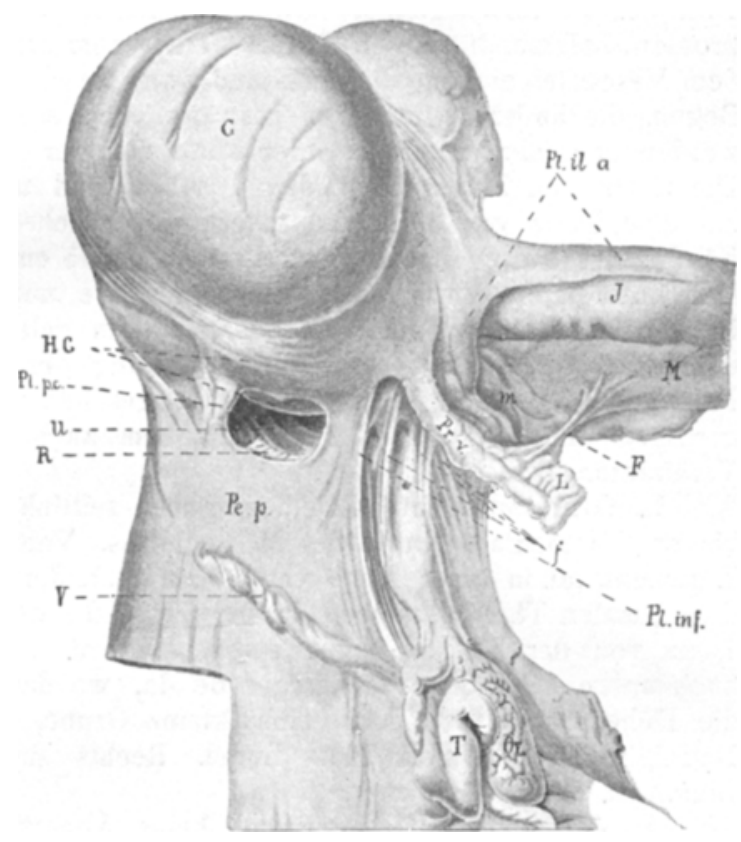
olum (m) eine kleine etwas sehnige, leicht silberglänzende Falte (F), deren grösste Höhenausdehnung etwa $1 / 2-1 \mathrm{~mm}$ beträgt, und zieht parallel dem freien Rande zum unteren Blatt des Ileumgekröses (M), wo sie sich, ohne den Darm ganz zu erreichen, verliert. Zur Plica ileo-appendicularis hat diese Falte keinerlei Beziehungen. Von dem unteren Blatt des Mesenteriolum ziehen mehrere sagittale Falten (f) nach der hinteren Bauchwand in ungleichmässigen Abständen, einander parallel, aber ungleich lang; die längste misst $2 \mathrm{~cm}$. Diese Falten bilden eine Art von Scheidewänden zwischen ungleich tiefen Nischen, Gefässe sind in ihnen nicht zu sehen. Die lateralste dieser Falten (Pl. inf.) ist die mächtigste, sie zieht von dem medialen Umfang des Coecum auf dem M. Psoas nach abwärts und ist identisch mit der Broesike'schen Plica infraangularis. Es zeigt sich nun weiter, dass sie mit der erwähnten ringförmigen Oeffnung hinter und unter dem Coecum 
in inniger Beziehung steht, insofern als der mediale Theil des Ringes direct in sie ubergeht.

Colon descend. und Flexura sigmoid. besitzen ein ca. $3-4 \mathrm{~cm}$ langes Mesocolon resp. Mesoflexur; auch deren Verlöthungslinie ist sehr deutlich dadurch, dass einerseite das Peritoneum des Mesocolon und der Mesoflexur viel feiner und durchsichtiger ist als das Peritoneum parietale, und dass andererseits in der Verlöthungslinie ein schmaler, silberglänzender Strang verläuft, der in etwas geschlängeltem Verlauf von dem noch zu erwähnenden Ansatzpunkt des Ligament. lat. nach oben zieht und auf diesem Wege verschiedene astförmige Ausläufer aussendet nach dem Peritoneum der hinteren Bauchwand speciell in einen Bezirk gerade am Beginn der Flexura sigmoid. Dieser Bezirk liegt zwischen zwei ziemlich grossen, halbmondförmigen Falten, welche vom parietalen Peritoneum nach dem Mesocolon ziehen, die eine, und zwar die obere, in abwärts concavem Bogen, die andere, untere, in aufwärts concavem Bogen. Beide Falten werden ungefähr gerade in ihrer Mitte von der Verlöthungslinie halbirt. Die unter den Falten liegenden Taschen sind ungleich tief, die obere hat eine Tiefe von $3 \mathrm{~cm}$, die untere eine solche von $2 \mathrm{~cm}$. Die freien Ränder der Falten haben einen Abstand von $5 \mathrm{~cm}$. Die obere der beiden Duplicaturen ist etwas mehr medial als die untere gelagert. An dem Peritoneum, das zwischen den beiden Falten gelegen ist, sind ebenso wie ober- und unterhalb dieses Bezirkes entlang der Verlöthungslinie kleine Falten und Vertiefungen vorhanden. Abgesehen von den schon erwähnten geringen Verdickungen ist das Peritoneum auch in diesem Theile ohne Veränderungen.

L. Ovar und Ostium tubae liegen oben seitlich vom Ureter und reichen bis an die mediale Kante des M. ileopsoas. Von hier erstreckt sich das Ligament. lat. in einer Höhe von $5 \mathrm{~cm}$ nach der Mesoflexur, um sich in dem basalen Theil derselben zu inseriren; die oberste Ausstrahlung liegt $7 \mathrm{~cm}$ von der Flexura sigm. selbst entfernt. Auf der medialen, etwas nach unten sehenden Fläche, gerade da, wo das Ligament. lat. sich in die Höhe erhebt, ist noch eine kleine Grube, in welcher gerade das Nagelglied des Fingers Platz findet. Rechts sind die Verhältnisse am Ligament. lat. die gleichen.

An den inneren Leistenringen keine Abnormität.

Brustsitus: Beide Lungen nicht retrahirt, sie stossen mit ihren Rändern in der Mittellinie zusammen. Vom Herzbeutel nur ein kleiner Theil sichtbar. Beide Pleurasäcke leer.

Herz von gewöhnlicher Grösse; linker Ventrikel gut contrahirt. Klappen sämmtlich gut durchgängig. Endocard fein und zart. Muskulatur links 11-12 mm dick, rechts 3-4 mm, von gewöhnlicher Farbe. In der Wand des linken Ventrikels mehrere gelbliche, wenig transparente Streifen. Mikroskopisch zeigt sich an Doppelmesserschnitten eine ziemliche starke, gleichmässige Verfettung in Gestalt kleiner Tröpfehen.

Zunge mit blasser Schleimbaut; an den Follikeln der Basis viele kleine Haemorrhagien.

Larynx und Trachea enthalten viel blutigen Schaum; ihre Schleimhaut leicht geröthet, stärker in den beiden Stammbronchien.

Lungen voluminös, uberall lufthaltig. Pleuraüberzug glatt und 
spiegelnd. Auf den Schnittfächen ist das Lungenparenchym hell und dunkelroth gefleckt, die dunkelrothen kleinen Flecken sind ein wenig prominent, aber nicht körnig, sondern glatt und spiegelnd wie die tbrigen Parthien. Das Gewebe lässt viel schaumige Flüssigkeit abstreichen und auspressen, es ist völlig zu compriminiren.

Art. und Vena pulmonal. frei.

Die Schleimhaut der kleinen und kleinsten Bronchien stark geröthet.

Die uibrigen Organe zeigen keine Veränderungen.

Auch die Darmschleimhant weist nur hier und da eine feine Injection der Gefässe auf. Ebenso ist die Schleimhaut des eingeklemmten Theiles normal bis auf eine schmale, circuläre Röthung, entsprechend dem eingezogenen Ring an der Aussenfläche.

Der bei der Section erhobene Befund war, wie wir gesehen haben, eine Bestätigung der Angaben des Operateurs. Während dieser aber nicht in der Lage war, über die specielle Art des Bruches Aufklärung zu geben, konnten wir feststellen, dass die Darmschlinge sich in einer hinter dem Coecum gelegenen Höhle verfangen haben musste. Wenn ich kurz noch einmal die Eigenschaften dieser Höhle recapitulire, wie sie sich nach dem Sectionsprotokoll darboten, und andererseits noeh einiges Weniges über das Ergebniss der Untersuchung an dem in Spiritus aufbewahrten Präparat hinzufïge, so ist also hervorzuheben, dass hinter dem Coecum eine ziemlich geräumige Höhle sich in leicht schräger Richtung nach oben erstreckte. Der Eingang zu dieser Höhle (cf. Abbild. R.) wurde von derbem Peritoneum gebildet, er war enger als die Höhle selbst und lag tief unter dem Blindsack des Coecum. Ein Zusammenhang mit der Plica infraangularis (Pl. inf.) ist deutlich nachzuweisen. Die Wandungen der Höhle werden gebildet hinten vom Peritoneum parietale der hinteren Bauchwand $(\mathbf{U})$, vorne im unteren Theil der Höhle von der Hinterwand des Coecum (H. C.), im oberen Theil, wo die Höhle nur noch zu einem Drittel hinter der Darmwand liegt und zu zwei Drittel lateral davon, wird die Vorderwand von einer Bauchfellduplicatur gebildet, die von der hinteren Bauchwand nach dem Coecum zieht und sich an demselben mit einer deutlich sichtbaren Verlöthungslinie inserirt. Im Verlauf dieser Verlöthungslinie befinden sich mehrere kleine Falten und Vertiefungen. Wir werden nicht irre gehen, wenn wir diese Bauchfellduplicatur als die Plica parieto-coecalis ansprechen, wenn sie auch die Eigenthümlichkeit besitzt, in ihrem unteren Theil stark verschmälert zu sein und mit einem sebr kurzen, freien Rande (Pl. p. c.) zu endigen, in welchem wir ein Analogon der sonst viel breiteren Plica parieto-coecalis zu erkennen glauben. Den medialen Abschluss der Höhle liefert die Verlöthungslinie des Coecum mit dem Peritoneum der hinteren Bauchwand, von welcher aus nach unten sich die Plica infraangularis fortsetzt. Wie 
sich nach dem Aufschneiden der hinteren Recessuswand feststellen liess, sind auch an dieser medialen Verlöthungslinie mehrfache kleine Vertiefungen und Falten vorhanden. Weiter liess sich an der eröffneten Höble noch constatiren, dass von ihrem Fundus aus zwei, mit ibrer Oeffnung gleichfalls in der Verlöthungslinie liegende, enge Kanäle hinter dem Coecum sich noch ungefähr $3 \mathrm{~cm}$ nach oben erstreckten. Der eine von ihnen lag medial und etwas tiefer als der andere, welcher ca. $1 \frac{1 / 2}{\mathrm{~cm}}$ von ihm in seitlicher Entfernung lag. Beide Canäle sind so eng, dass man nur eine feine Sonde in sie einführen kann.

Die normale Beschaffenheit des den Recessus auskleidenden Peritoneum, welche vorher nur mit dem Tastgefühl constatirt werden konnte, wurde jetzt durch den Augenschein bestätigt.

Nach dem Gesagten dürfte es wohl nicht zweifelhaft erscheinen, dass unser Recessus mit dem Recessus retrocoecalis, wie Broesike ihn geschildert hat, völlig übereinstimmt. Weiter scheint auch kein Zweifel darüber möglich, dass diese Höhle es war, welche für die eingeklemmte Darmschlinge sowohl Bruchpforte als Bruchsack abgab. Wollte man den Aussagen des Operateurs und dem Mangel einer anderen Bruchpforte nicht trauen, so würde doch der Umstand, dass ausser am Schnürringe des Darmes nur am Eingange zu dem Recessus sich entzündliche Erscheinungen darboten, wohl als genügend beweiskräftig gelten können. Damit würde unser Fall als zweiter, sicher gültiger Fall einer Hernie des Recessus retrocoecalis in die Casuistik einzureihen sein. Er gestattet uns nicht, wie der $A$ sch off'sche, einen Schluss auf die Genese solcher IIernien zu ziehen, ebensowenig wie er es möglich macht, über die speciellen klinischen Erscheinungen und die eventuelle Diagnostik solcher Hernien des Recessus retrocoecalis ein für sie charakteristisches Bild zu entwerfen. Spätere Untersucher, die vielleicht das Glück haben werden, solche Fälle von Beginn an beobachten zu können, werden entscheiden müssen, ob es überhaupt möglich ist, bei solchen Hernien eine anatomisch genau localisirte Diagnose zu stellen, speciell ob sie differentialdiagnostisch von den Hernien des Recessus ileoappendicularis zu unterscheiden sind.

Noch einiger Worte bedürfen jene beiden grossen Falten und Taschen am unteren, linksseitigen Blatt der Mesoflexur. Auch sie scheinen gerade so wie die kleinen Falten und Vertiefungen im Verlauf der Verlöthungslinie des Colon descend. und der Flexura sigmoid., deren Analoga sich auch rechts an der Verlöthungslinie des Darmes fanden, durch Unregelmässigkeiten im Verlöthungsprocess entstanden 
zu sein. Denn auch in der Verlöthung des Colon descend. und der Flexura sigmoid. kommen die grössten Varietäten vor, wie besonders Broesike gelegentlich der Besprechung des Recessus intersigmoideus hervorhebt. In dem Spalteholz'schen Atlas findet sich eine $\mathbf{A b}$ bildung, die jene kleinen Vertiefungen in grosser Zahl lateral vom Colon descend. darstellt, Spalteholz nennt sie dort Recessus paracolici. Ich habe solche Bildungen mehrfach bei Individuen aller Lebensalter, besonders aber des Kindesalters, gesehen.

Praktische Bedeutung scheint diesen Taschen, auch wenn sie so gross sind wie in unserem Falle, wohl kaum zuzukommen.

Ehe ich schliesse, ist es mir ein aufrichtiges Bedürfniss, meinem hochverehrten Chef, Herrn Prof. Lang hans, für sein reges Interesse und die thatkräftige Förderung, die er dieser Arbeit besonders durch Unterstützung mit der einschlügigen Literatur hat zukommen lassen, meinen herzlichsten Dank zu sagen.

\section{Literaturverzeichniss.}

1) Broesike, Ceber intraabdominale (retroperitoneale) Hernien und Bauchfelltaschen nebst einer Darstellung der Entwickelung peritonealer Formationen. Berlin 1891.

2) Jonnesco, Hernies internes rétro-péritonéales. Paris 1890.

3) A b bée, Ucber Hernia duodenojejunal. Ziegler's Beiträge z. patholog. Anatomic u. z. allgem. Patholog. Bd. 29. 1901.

4) M a nki, Ueber Rotroperitonealhernien. Münch. med. Wochenschr. 1896.

5) Bingel, Ueber Hernia retroperitonealis duodenojejunalis (Treitzii). Virch. Arch. Bd. 167. S. 125 .

6) Waldeyer, Hernia retroperitonealis nebst Bemerkungen zur Anatomie der Peritonitis. Virch. Arch. Bd. 60. 1874.

7) Luschka, Anatomie des Banches. - Leber die peritoneale Linhüllung des Blinddarms und über die Fossa ileo-coecal. Virch. Arch. Bd.21. 1861. Die organische Musculatur verschiedener Falten des menschlichen Bauchfells. Arch. f. Anat. 1. Physiol. 1862.

8) Merkel, Handbuch d. topograph. Anatomie. 1899.

9) Spalteholz, Anatom. Atlas.

10) Toldt, Bau und Wachsthumsverhältnisse der Gekröse des menschlichen Darmkanals. Denkschriften der kais. Akad. d. Wissenschaften zu Wien. Febr. 1579. Bd. 41. - Zur Charakterist. und Entstehung des Recess. duodenojejunal. Prager med. Wochenschr. 1879. - Die Darmgekröse und Netze im gesetzmässigen und gesetzwidrigen Zustande. Denkschr. d. kais. Akad. d. Wissensch. Math. naturwiss. Classe. Bd, 56. 1859. - Anatomischer Atlas.

11) Snow, London. med. Gazette. 1846.

12) Nasse, Fin Fall von incarcerirter Hernia ileoappendicular. Archiv f. klin. Chirurgie. Bd. 51. 
$516 \mathrm{XXX}$. Funkensteds, Ein Beitrag zur Lehre von den intraabdominalen Hernien.

13) Riese, Ueber einen durch Laparotomie geheilten Fall von Hernia ileo-coecal. incarcerata. Arch. f. klin. Chirurgie. Bd. 60. 1900.

14) Huschke, Lehre von den Eingeweiden and Sinnesorganen des menschlichen Körpers. Leipzig 1844.

15) Treitz, Hernia retroperitonealis. Ein Beitrag zur Geschichte innerer Hernien. Prag 1857.

16) Tarenetzki, Beiträge zur Anatomie des Darmkanals. Mémoires de l'Acad. d. St. Petersbourg. Serie VII. T. 28. 1881.

17) Asch off, Zur Lehre von der Darmeinklemmung in innere Bauchfelltaschen. Berl. Klinik. Heft 100. 1896.

18) Rieux, Considérations sur l'étranglement de l'intestin dans la cavité abdominale et sur un mode d'étranglement non décrit par les auteurs. Thèse de Paris. 1853.

19) Engel, Anatom. Mittheilungen für die Praxis. Wien. med. Wochenschr. 1857.

\section{Erklärung der Abbildung.}

Die Zeichnung zeigt den Eingang zu dem Recessus retrocoecalis, sie ist nach dem in Spiritus aufbewahrten Präparat in $1 / 2$ natürlicher Grösse angefertigt.

C Blindes Ende des Coecum, stark gefüllt und etwas nach oben gehoben.

H. CHinterwand des Coecum, welche als schmaler Saum noch in dem Recessus zu sehen ist.

$R \quad$ Schwellenförmige Erhebung des verdickten Peritoneum parietale.

$U$ Hinterwand des Recessus aus normalem Peritoneum pariet. bestehend.

Pe. p. Peritoneum parietale der hinteren Bauchwand.

$P l$. p. c. Stark verkürzte Plica parieto-coecalis.

$P l$. inf. Kante der Plica infraangularis.

$I \quad$ Ileum.

Pl. il. a. Fettreiche, daher etwas wulstige Plica ileo-appendicularis.

$M$ Hinteres unteres Blatt des Mesenterium.

$m$ Mesenteriolum des Process. vermiform. (Pr.v.).

$L \quad$ Fettläppchen am Process. vermiformis.

$F \quad$ Falte im Mesenteriolum parallel dem freien Rande.

$f$ Kleine sagittale Falten vom unteren Blatt des Mesenteriolum ausgehend parallel der Plica infraangularis.

Ov. Ovarium.

$T$ Tube.

$V \quad$ Falte im Peritoneum parietale, bei der Conservirung entstanden. 